
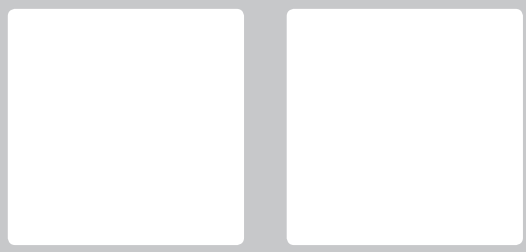

\title{
FUSÕES E AQUISIÇÕES NA INDÚSTRIA MANUFATUREIRA: UMA ANÁLISE ECONOMÉTRICA DAS COMPANHIAS DE CAPITAL ABERTO
}

\section{Mergers and Acquisitions in the Manufacturing Industry: an econometric analysis of publicly traded companies}

\section{Thaís Alves dos Santos \\ Minicurrículo}

e-mail:autor1@XXX.com

\section{Cristina Lelis Leal Calegario}

Minicurrículo

e-mail:autor2@XXX.com

\section{Francisval de Melo Carvalho}

Minicurrículo

e-mail:autor2@XXX.com

\section{RESUMO}

O principal objetivo deste estudo foi investigar os fatores determinantes das operações de Fusão e aquisição (F\&A) na Indústria Manufatureira, sob a ótica das empresas adquirentes. A natureza do estudo é quantitativa e a verificação dos fatores determinantes das F\&A aconteceu por meio de um modelo de regressão logística. O período de análise deste trabalho compreende os anos de 2001 a 2011. Dentre as várias características das empresas estudadas foi possível identificar algumas que se confirmaram determinantes para os processos de F\&A. Pode-se afirmar que com base nos resultados que as características da empresa que são determinantes das operações de F\&A são a eficiência operacional, rentabilidade, retorno ao acionista e o tamanho da empresa. Concluiu-se que os resultados deste trabalho são relevantes, pois foi possível identificar alguns fatores determinantes das F\&A.

Palavras-chave: fusões e aquisições, regressão logística, indústria manufatureira.

\section{ABSTRACT}

The main objective of this study was to investigate the determinants of Merger and Acquisition (M\&A) operations in the Manufacturing Industry, from the perspective of the acquiring companies. The nature of the study is quantitative and the verification of the determinants of M\&A happened through a logistic regression model. The period of analysis of this work covers the years from 2001 to 2011. Among the several characteristics of the companies studied, it was possible to identify some that confirmed the determinants of M\&A processes. It can be said that based on the results that the characteristics of the company that are determinants of M\&A operations are the operational efficiency, profitability, return to the shareholder and the size of the company. It was concluded that the results of this work are relevant, since it was possible to identify some determinant factors of M\&A.

Keywords: mergers and acquisitions, logistic regression, manufacturing industry. 


\section{INTRODUÇÃO}

As transformações econômicas globais ditam o comportamento dos diversos atores da economia. A intensidade da integração da economia proporciona reflexos instantâneos no ambiente de negócios, tanto em momentos de recessão como nos de ascensão econômica.

Todas essas transformações na economia nacional auxiliaram a ampliação do número de transações de fusões e aquisições (F\&A). As empresas nacionais e estrangeiras vislumbram nessas operações oportunidades de crescimento, ademais, tais operações podem ser consideradas resultado das estratégias da empresa (ÖBERG; HOLTSTRÖM, 2006).

Contudo o crescimento do número de operações de F\&A no país, somente foi possível por meio da desregulamentação dos mercados locais, pelo interesse de empresas estrangeiras nos mercados emergentes e a permissão de compra (dos governos) de empresas nacionais por estrangeiras. Nesse cenário, as F\&A ocupam papel de destaque, enquanto estratégia de crescimento e sobrevivência das firmas.

É importante destacar que as operações de F\&A, no Brasil, em 2011, cresceram 12,5\% em relação a ano anterior. Desde então os números têm sido crescentes, registrando um aumento de 12\% em 2017 se comparado com o ano de 2016. Sendo que, todas as operações envolveram direta ou indiretamente empresas brasileiras (KPMG, 2012 e 2018).

No anseio de identificar as causas e benefícios das operações de F\&A, diversos estudos observam as características financeiras e do mercado acionário antes e depois da efetivação dos processos (CAMERLYNCK; OOGHE; LANGHE, 2005; CLEMENTS; SING, 2011; DENČIĆ-MIHAJLOV; RADOVIĆ, 2006; PALEPU, 1986; BARNES, 1998; DUCHIN e SCHMIDT, 2013; TOIGO e HEIN, 2017; HSU; WRIGHT e ZHU, 2017).

A partir da relevância do tema e no anseio de compreender melhor como estas operações se dão, o principal objetivo deste trabalho foi identificar quais são os fatores que determinam as operações de F\&A nas empresas de capital aberto da Indústria Manufatureira no Brasil. Este recorte, Indústria Manufatureira, se deu, entre outros, pois a mesma comporta cerca de dez setores distintos da economia brasileira, como siderurgia e metalurgia, alimentos e bebidas, petróleo e gás, máquinas industriais, eletroeletrônico, têxtil, veículos e peças, química, dentre outros, isto é, o trabalho envolve diversos setores da economia. Ademais, o desenvolvimento das economias passa pelo crescimento do setor industrial, uma vez que, o aumento da participação deste no Produto Interno Bruto (PIB) de um país contribui positivamente para o crescimento da economia (CHENERY, 1960; VIEIRA; AVELLAR e VERÍSSIMO, 2014). No Brasil, resultados da Pesquisa Industrial Anual (PIA) apontam para a importância da indústria na economia nacional (IBGE, 2010 e 2016).

Portanto, para cumprir o objetivo de identificar os fatores determinantes das operações de F\&A este trabalho utilizou como técnica estatística a regressão logística. Posto que a regressão logística permite ao pesquisador trabalhar com variáveis qualitativas e assim conseguir identificar quais variáveis dependentes são determinantes para o acontecimento do fato estudado, neste caso, as F\&A.

A fim de subsidiar o entendimento, apresenta-se uma revisão de fundamentos teóricos relacionados aos conceitos das operações de F\&A, posteriormente, apresenta-se a descrição da metodologia empregada para a investigação empírica do problema e, por fim, são apresentados os resultados dos métodos utilizados e as conclusões finais

\section{REFERENCIAL TEÓRICO}

\subsection{Fusões e aquisições: conceitos, motivações e ondas}

Tradicionalmente há duas formas de estratégias empresariais para o crescimento: o crescimento interno e o externo. O primeiro significa que o crescimento da empresa se dá por meio dos recursos e pontos fortes das empresas. O segundo seria o crescimento por meio da compra ou associação com outra empresa ou negócio, ou seja, operações de F\&A (PARK; JANG, 2011).

As F\&A são, na maioria das vezes, tratadas de maneira indistinta (DHAWAN, 2009). No entanto, consistem em práticas distintas, por isso, é importante diferenciar os conceitos de fusão e aquisição. As 
operações de F\&A podem ser definidas como uma estratégia na qual, duas empresas se aliam, integrando suas operações, pois juntas conseguem ampliar suas vantagens competitivas (KLOECKNER, 1994; TRICHES, 1996; WESTON; SIU; JOHNSON, 2006; PEREIRA; DORNELAS, 2010; GAUGHAN, 2011). Por sua vez, a Lei $n^{\circ} 6404 / 76$ e posteriores alterações, conhecida por Lei das Sociedades Anônimas, apresenta os seguintes conceitos de fusão e aquisição, respectivamente:

- é a operação pela qual se unem duas ou mais sociedades para formar uma sociedade nova, que lhes sucederá em todos os direitos e obrigações (art. 228)

- quando uma das sociedades compra outra, sucedendo-a em todos seus direitos e obrigações (art. 227).

Assim, conforme ressaltado, fusão é uma combinação de, ao menos duas empresas, em que uma terceira empresa será criada como resultado desse acordo. Aquisição é a compra de uma empresa por outra em que, geralmente, a adquirente é maior que a empresa alvo. Ambos os processos envolvem troca financeira, seja de ações ou o pagamento de determinado valor estipulado em negociação e, normalmente, acontecem de forma voluntária (TRICHES, 1996; WESTON; SIU; JOHNSON, 2006; DHAWAN, 2009; GAUGHAN, 2011).

As fusões podem ser classificadas em: (i) horizontal quando estão envolvidas duas empresas, que competem em mercados iguais, objetivando o ganho de vantagens específicas de economias de escala e escopo; (ii) vertical quando envolve firmas em diferentes estádios de produção, com o objetivo de absorver os processos sequenciais ao longo da cadeia produtiva que, anteriormente, eram negociados via mercado e (iii) conglomerado quando abarca empresas em atividades e negócios não relacionados entre si, fora de seu âmbito de atuação (PIMENTEL, 2004; WESTON; SIU; JOHNSON, 2006; GAUGHAN, 2011).
No que tange à aquisição, há três modalidades básicas: (i) fusão ou consolidação, (ii) aquisição de ações e (iii) aquisição de ativos. A aquisição de ações ocorre quando uma empresa compra as ações, com direito a voto, de outra empresa e a aquisição de ativos quando a empresa compra todos os ativos de outra empresa, porém, para tal, é necessário que os acionistas da empresa vendedora aprovem formalmente a operação (ROSS; WESTERFIELD; JAFFE, 2007; TRICHES, 1996).

Diversos fatores macroeconômicos podem gerar uma atividade de F\&A, como o crescimento do PIB do país, a taxa de juros e as políticas monetárias. $\mathrm{O}$ ritmo crescente dos movimentos de F\&A pode ser justificado pelas mudanças relevantes que acontecem no cenário econômico, em âmbito macro e microeconômico. A desregulamentação da economia, os avanços tecnológicos, as peculiaridades das indústrias em geral compõem as principais mudanças que alimentam esses processos (WESTON; SIU; JOHNSON, 2006; DHAWAN, 2009).

Assim, no decorrer dos anos, observa-se períodos de atividades intensas das operações de F\&A, alguns autores as classificam em ondas (CAMARGOS; BARBOSA, 2003; WESTON; SIU; JONHSON, 2006; GAUGHAN, 2011). A classificação das operações de F\&A em ondas ocorre, em sua maioria, em função das atividades de F\&A nos Estados Unidos. Contudo, não há consenso em relação ao número de ondas de F\&A. Scherer (1970) afirma que existiram três ondas, Weston, Siu e Johnson (2006) as dividem em cinco ondas e Gaughan (2011) apresenta em seu trabalho seis ondas de F\&A, como observado no Quadro 1.

Pessanha (2016) e Hunjra et al (2014) ainda afirmam que os processos de F\&A são acontecimentos de relevância no mercado internacional. Podendo ser considerado um processo de resposta ao grau de incerteza dos mercados, resultados da atuação das empresas em diversas economias, e, consequente acirramento da concorrência. 
Quadro 1 Ondas de fusão e aquisição

\begin{tabular}{|c|c|c|}
\hline Autor(es) & Onda & Período \\
\hline \multirow[t]{3}{*}{ Scherer (1970) } & The Great Merger Wave & 1887-1904 \\
\hline & The Merger Movement & 1916-1929 \\
\hline & The Post World War II Merger Movement & Posterior a 1945 \\
\hline \multirow[t]{5}{*}{ Weston, Siu e Johnson (2006) } & 1a Onda & 1895-1904 \\
\hline & $2^{\mathrm{a}}$ Onda & 1922-1929 \\
\hline & 3a Onda & 1940-1947 \\
\hline & $4^{\mathrm{a}}$ Onda & Década de 1960 \\
\hline & $5^{\mathrm{a}}$ Onda & A partir de 1976 \\
\hline \multirow[t]{6}{*}{ Gaughan (2011) } & 1a Onda & 1897-1904 \\
\hline & 2 a Onda & 1916-1929 \\
\hline & 3a Onda & 1965-1969 \\
\hline & 4a Onda & 1984-1989 \\
\hline & 5a Onda & $1992-2000$ \\
\hline & 6a Onda & $2004-2007$ \\
\hline
\end{tabular}

\subsection{Estudos brasileiros de fusão e aquisição}

Historicamente os processos de F\&A são mais acentuados em economias consolidadas como a dos Estados Unidos e Europa. Todavia as operações de F\&A apresentam números crescentes no ambiente de negócios do Brasil (MIRANDA; MARTINS, 2000; WOOD JR; VASCONCELOS; CALDAS, 2004; PESSANHA, 2016).

Para Wood Jr., Vasconcelos e Caldas (2004), assim como em muitos países emergentes, $\mathrm{o}$ aumento das operações de F\&A no Brasil acompanhou o processo de liberalização econômica a partir da década de 1990. Para o autor:

esse fator favoreceu os processos de F\&A de três diferentes modos: primeiro, a desregulamentação dos mercados locais, associada às tendências internacionais em direção à globa- lização, permitiu que as empresas estrangeiras adquirissem empresas brasileiras; segundo, programas de privatização criaram oportunidades para que muitas empresas estrangeiras e brasileiras adquirissem grandes operações nos setores de energia, de telecomunicações e bancário; e terceiro, a elevada competição internacional, associada à acelerada mudança tecnológica, obrigou empresas domésticas a se fundirem ou a adquirirem umas às outras.

Estas características da década de 1990, corroboram com Triches (1996), que afirma que esta década tem como característica a versatilidade de que as empresas buscassem novos arranjos como forma de se adaptar ao novo ambiente de negócios e entrar em novos mercados. Assim, o Quadro 2 apresenta alguns trabalhos de autores brasileiros que estudaram os processos de F\&A. É interessante observar que alguns trabalhos apresentam resultados empíricos contraditórios, como por exemplo os resultados dos trabalhos de Franco e Camargos (2011) e o trabalho de Pessanha et al. (2012b). 
Quadro 2 Estudos brasileiros de F\&A

\begin{tabular}{|c|c|c|c|}
\hline Autor & Objetivo & Metodologia & Considerações/Conclusões \\
\hline $\begin{array}{l}\text { Zilber, Fichmann e } \\
\text { Pikieny (2002) }\end{array}$ & $\begin{array}{l}\text { Identificar a utilização da } \\
\text { F\&A como estratégia de } \\
\text { crescimento. }\end{array}$ & $\begin{array}{l}\text { Estudo exploratório com } \\
\text { entrevistas em profundi- } \\
\text { dade }\end{array}$ & A F\&A foi utilizada como estratégia de crescimento. \\
\hline $\begin{array}{l}\text { Wood Jr., Vasconcelos } \\
\text { e Caldas (2004) }\end{array}$ & $\begin{array}{l}\text { Identificar objetivos e } \\
\text { consequências das F\&A } \\
\text { no Brasil }\end{array}$ & $\begin{array}{l}\text { Entrevistas com executivos } \\
\text { e gerentes das empresas }\end{array}$ & $\begin{array}{l}\text { - reação/antecipação aos concorrentes; } \\
\text { - o resultado mais concreto foi o aumento de porte; } \\
\text { - condução do processo gera impactos no resultado } \\
\text { final; } \\
\text { - problemas de agência podem dificultar os processos. }\end{array}$ \\
\hline $\begin{array}{l}\text { Brito, Batistella e Famá } \\
\text { (2005) }\end{array}$ & $\begin{array}{l}\text { Verificar a criação de valor } \\
\text { ao acionista da empresa } \\
\text { adquirente. }\end{array}$ & Estudo de eventos & $\begin{array}{l}\text { As operações de F\&A não proporcionaram aumento } \\
\text { de riqueza aos acionistas das empresas adquirentes. }\end{array}$ \\
\hline $\begin{array}{l}\text { Patrocínio, Kayo e } \\
\text { Kimura (2007) }\end{array}$ & $\begin{array}{l}\text { Analisar a relação entre } \\
\text { intangibilidade e criação } \\
\text { de valor em F\&A }\end{array}$ & Estudo de eventos & $\begin{array}{l}\text { F\&A que envolvem empresas com intangível-intensi- } \\
\text { vas apresentaram } \\
\text { um potencial de retorno significativamente maior } \\
\text { que as operações que envolvera m empresas tangí- } \\
\text { vel-intensivas. }\end{array}$ \\
\hline $\begin{array}{l}\text { Camargos e Barbosa } \\
\text { (2009) }\end{array}$ & $\begin{array}{l}\text { Verificar a criação de siner- } \\
\text { gias operacionais e criação } \\
\text { de valor. }\end{array}$ & $\begin{array}{l}\text { Estatística descritiva - com- } \\
\text { paração de médias. }\end{array}$ & $\begin{array}{l}\text { As operações de F\&A proporcionam sinergias opera- } \\
\text { cionais para as empresas e conduz à maximização do } \\
\text { valor de mercado gerando riqueza aos acionistas. }\end{array}$ \\
\hline $\begin{array}{l}\text { Franco e Camargos } \\
\text { (2011) }\end{array}$ & $\begin{array}{l}\text { Verificar se os processos } \\
\text { de F\&A geram sinergias } \\
\text { operacionais, aumento na } \\
\text { rentabilidade e criação de } \\
\text { valor para os acionistas. }\end{array}$ & $\begin{array}{l}\text { Estatística Descritiva - } \\
\text { comparação de médias }\end{array}$ & $\begin{array}{l}\text { Existe a criação e valor, aumento na rentabilidade e as } \\
\text { sinergias operacionais foram positivas. }\end{array}$ \\
\hline Nardi e Silva (2012) & $\begin{array}{l}\text { A condição do mercado } \\
\text { (mercado em alta ou baixa) } \\
\text { influencia na criação de } \\
\text { valor em F\&A. }\end{array}$ & $\begin{array}{l}\text { Retorno anormal acumu- } \\
\text { lado. }\end{array}$ & $\begin{array}{l}\text { Sem evidências suficientes para afirmar se o senti- } \\
\text { mento do mercado influência na criação e valor. }\end{array}$ \\
\hline Pessanha et al. (2012a) & $\begin{array}{l}\text { Verificar o impacto das F\&A } \\
\text { na rentabilidade. }\end{array}$ & Modelos de intervenção. & $\begin{array}{l}\text { As F\&A foram consideradas uma intervenção positiva } \\
\text { na rentabilidade das empresas. }\end{array}$ \\
\hline Pessanha et al. (2012b) & $\begin{array}{l}\text { Existe a criação de valor } \\
\text { para acionistas nas opera- } \\
\text { ções de F\&A. }\end{array}$ & Estudo de eventos. & Não existe a criação de valor. \\
\hline $\begin{array}{l}\text { Duchin e Schmidt } \\
\text { (2013) }\end{array}$ & $\begin{array}{l}\text { Como são as operações } \\
\text { de F\&A durante as ondas } \\
\text { de F\&A. }\end{array}$ & $\begin{array}{l}\text { Buy and hold abnormal } \\
\text { returns (BHAR) }\end{array}$ & $\begin{array}{l}\text { Concluíram que as operações de F\&A que ocorrem } \\
\text { durante as ondas de F\&A apresentam resultados } \\
\text { aquém dos esperados. Ressaltam que a governança } \\
\text { corporativa é fraca nesses períodos e por isso podem } \\
\text { ser influenciadas por problemas de agência. }\end{array}$ \\
\hline Bortoluzzo et al (2014) & $\begin{array}{l}\text { Verificar se empresas } \\
\text { nacionais que realizam pro- } \\
\text { cessos de F\&A cross border } \\
\text { têm melhores resultados } \\
\text { financeiros }\end{array}$ & $\begin{array}{l}\text { Regressão com dados em } \\
\text { painel }\end{array}$ & $\begin{array}{l}\text { Concluíram que os processos de F\&A cross border } \\
\text { resultaram em melhor desempenho financeiro para } \\
\text { as empresas que utilizaram desta prática. }\end{array}$ \\
\hline Pessanha (2016) & $\begin{array}{l}\text { Identificar a existência de } \\
\text { padrões de ocorrência } \\
\text { nos processos de F\&A de } \\
\text { instituições financeiras }\end{array}$ & $\begin{array}{l}\text { Redes neurais artificiais } \\
\text { (RNA), análise discriminan- } \\
\text { te linear ( } A D L) \text { e regressão } \\
\text { logística (RL) }\end{array}$ & $\begin{array}{l}\text { Concluiu que as características econômico-financeira } \\
\text { das empresas, juntamente com as características do } \\
\text { mercado e economia em que as empresas operam } \\
\text { são decisivas para os processos de F\&A }\end{array}$ \\
\hline
\end{tabular}

Apesar de se encontrar resultados contraditórios em alguns trabalhos empíricos, a grande maioria dos trabalhos usam de informações financeiras para avaliar os retornos financeiros e a criação de valor 
dessas operações. King et al. (2004) afirmam que "a lógica teórica mais comum para F\&A é a busca por sinergia, ou o conceito que a soma das duas firmas em processo de fusão é maior que suas partes individuais (p.ex. $2+2=5$ )." A falta de uma teoria geral que alicerce os processos de F\&A pode contribuir para estes resultados contraditórios (KLOECKNER, 1994).

\subsection{Modelo teórico}

O modelo teórico deste trabalho foi construído para identificar os fatores determinantes das operações de F\&A. O foco deste está nas empresas que realizaram operações de F\&A, o que os teóricos chamam de empresas adquirentes.
Partindo do pressuposto que as operações de F\&A alteram a estrutura da firma e do mercado em que as empresas estão inseridas (KUPFER; HASENCLEVER, 2002; CAMARGOS; BARBOSA, 2003), foram selecionadas variáveis que refletem a situação econômico-financeira, alguns índices econômicos financeiros das empresas.

As variáveis utilizadas no modelo são variáveis baseadas nos trabalhos de Palepu (1986), Barnes (1998 e 1999), Camargos e Barbosa (2003), Denčić-Mihajlov e Radović (2006), Brar, Giamouridis e Liodakis (2009), Bauguess et. al. (2009) e Franco e Camargos (2011) representadas na Figura 1. A composição das variáveis pode ser observada no Quadro 6 no tópico 3.2 deste trabalho.

Figura 1 Modelo teórico e variáveis

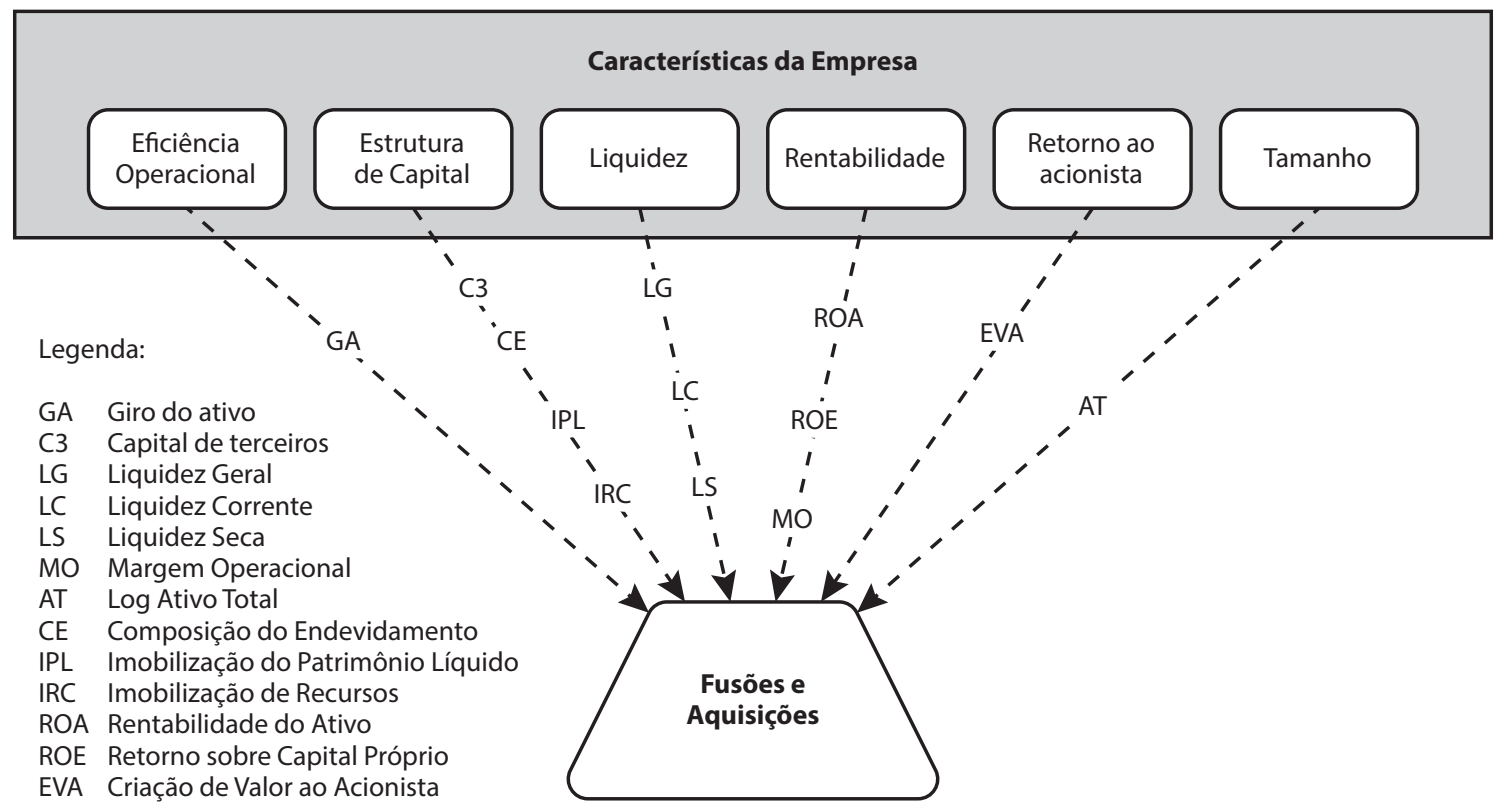

\section{ASPECTOS METODOLÓGICOS}

\subsection{Tratamento e origem dos dados}

A amostra deste estudo foi composta por empresas que realizaram ou não operações de fusões e aquisições, no período temporal de 2001 a 2011, com dados financeiros trimestrais. O recorte temporal teve como objetivo utilizar o período anterior ao novo regimento do Cade (Conselho Administrativo de De- fesa Econômica), em vigor a partir de maio de 2012. Os dados foram organizados em painel balanceado.

Utilizando a classificação setorial NAICS (North American Industry Classification System) a Indústria Manufatureira foi selecionada para este trabalho. A escolha deste setor se deu, pelo mesmo apresentar um número considerável de empresas ativas, ou seja, com negociação em bolsa, e com dados financeiros disponíveis, além de se considerar a relevância do mesmo na economia nacional. Assim a amostra inicial foi 
composta por cento e trinta e cinco empresas. Os dados financeiros destas empresas foram analisados e as empresas que não apresentaram informações disponíveis no período proposto foram retiradas da amostra. Desta forma a amostra final é formada por oitenta e seis empresas deste setor. Estas foram divididas em dois grupos distintos: um de empresas que realizaram operações de F\&A (Quadro 3) e o outro das empresas que não realizaram F\&A (Quadro 4).

Quadro 3 Empresas com F\&A

\begin{tabular}{|c|c|c|c|}
\hline Empresa & $N^{\circ} \mathbf{F} \& A$ & Empresa & No F\&A \\
\hline Alpargatas S.A. & 4 & Klabin S. A. & 1 \\
\hline Cia de Bebidas das Américas - Ambev & 10 & M G Poliester S. A. & 1 \\
\hline Bardella S. A. Indústrias Mecânicas & 1 & Mangels Industrial S. A. & 1 \\
\hline Bombril S. A. & 1 & Marcopolo S. A. & 1 \\
\hline BRF - Brasil Foods S. A. & 14 & Mahle-Metal Leve S. A. & 1 \\
\hline Celulose Irani S. A. & 1 & Minupar Participações S. A. & 1 \\
\hline Elekeiroz S. A. & 1 & Nadir Figueiredo Ind e Com S. A. & 1 \\
\hline Eternit S. A. & 1 & Plascar Participações Industriais S. A. & 3 \\
\hline Forjas Taurus S. A. & 1 & Randon S. A. Implementos e Participações & 1 \\
\hline Fras-Le S. A. & 1 & Rimet Empreendimentos Industriais e Comerciais S. A. & 1 \\
\hline Haga S. A. Indústria e Comércio & 1 & Schulz S. A. & 1 \\
\hline Cia Iguaçu de Café Solúvel & 1 & Cia Siderúrgica Nacional & 11 \\
\hline Indústrias Romi S. A. & 2 & Suzano Papel e Celulose S. A. & 3 \\
\hline Inepar S. A. Indústria e Construções & 1 & Ultrapar Participações S. A. & 1 \\
\hline lochpe Maxion S. A. & 6 & Unipar Participações S. A. & 1 \\
\hline Itautec S. A. - Grupo Itautec & 1 & Usinas Sid de Minas Gerais S. A. - Usiminas & 1 \\
\hline Karsten S. A. & 1 & Weg S. A. & 1 \\
\hline
\end{tabular}

Os dados referentes as informações econômico-financeiras das empresas (dados trimestrais) foram coletadas no banco de dados Economática․ Já as informações sobre as operações de F\&A de cada empresa foram obtidas por meio de busca nos relatórios de administração, comunicados ao mercado e fatos relevantes das empresas da amostra, divulgados na Comissão de Valores Mobiliários (CVM), Bolsa, Brasil, Balcão (B3) e sites de relacionamento dos com os investidores da empresa.
Para identificar os fatores que determinam as operações de F\&A das empresas foi necessário trabalhar com modelos para variáveis discretas, isto é, aqueles que utilizam variáveis qualitativas como variável dependente. Uma vez que se trata da ocorrência ou não dos processos de F\&A. Assim, o modelo escolhido foi o modelo de regressão logística, também conhecido como modelo logit binário. 
Quadro 4 Empresas sem operações de F\&A

\begin{tabular}{|c|c|}
\hline \multicolumn{2}{|c|}{ Empresas sem operações de F\&A } \\
\hline Electro Aco Altona S. A. & Metalúrgica Duque S. A. \\
\hline Siderúrgica J. L. Aliperti S. A. & Metalgráfica Iguacu S. A. \\
\hline Baumer S. A. & Metisa Metalúrgica Tmboense S. A. \\
\hline Bicicletas Monark S. A. & Millennium Inorganic Chemicals BR S. A. \\
\hline Buettner S. A. Indústria e Comércio & Conservas Oderich S. A. \\
\hline Cia Cacique de Café Solúvel & Panatlantica S. A. \\
\hline Café Solúvel Brasília S. A. & Paranapanema S. A. \\
\hline Cia Fiação Tecidos Cedro Cachoeira & Refinaria de Petróleos Manguinhos S. A. \\
\hline Cia Hering & Pettenati S. A. Indústria Textil \\
\hline Cobrasma S. A. & Portobello S. A. \\
\hline Cia Tecidos Norte de Minas Coteminas & Recrusul S. A. \\
\hline Dohler S. A. & Metalúrgica Riosulense S. A. \\
\hline Empresa Nac. Com. Redito Part. S. A. Encorpar & Sansuy S. A. Indústria de Plásticos \\
\hline Manufatura de Brinquedos Estrela S. A. & Cia Tecidos Santanense \\
\hline Eucatex S. A. Indústria e Comércio & Cia Industrial Schlosser S. A. \\
\hline Excelsior Alimentos S. A. & Souza Cruz S. A. \\
\hline Fábrica de Tecidos Carlos Renaux S. A. & Springer S. A. \\
\hline Ferbasa & Tectoy S. A. \\
\hline Fibam Companhia Industrial & Tekno S. A. - Indústria e Comércio \\
\hline Gerdau S. A. & Textil Renauxview S. A. \\
\hline Guararapes Confecções S. A. & Tupy S. A. \\
\hline Hercules S. A. Fábrica de Talheres & Vicunha Textil S. A. \\
\hline Cia Industrial Cataguases & Wembley Sociedade Anônima \\
\hline Josapar-Joaquim Oliveira S. A. - Particip & Wetzel S. A. \\
\hline Kepler Weber S. A. & Whirlpool S. A. \\
\hline Cia Melhoramentos de São Paulo & \\
\hline
\end{tabular}

Para Field (2009) a regressão logística pode ser resumidamente definida como uma regressão múltipla com variável de saída categórica dicotômica (discreta) e variáveis previsoras contínuas ou categóricas. Hair Jr. et al. (2009) afirmam que a variável estatística da regressão logística, assim como na regressão múltipla, representa uma relação multivariada com os coeficientes, ou seja, indica qual o impacto relativo de cada variável preditora.
O modelo logit foi especificamente elaborado para prever a probabilidade de um evento ocorrer. No caso deste trabalho, qual a chance de que cada característica econômico-financeira da empresa levantada pertença ao grupo das empresas que realizaram processos de F\&A, indicando que a mesma é determinante para este processo.

O modelo logit, cuja variável dependente Y é binária, pode ser representado por (STOCK; WATSON, 2004): 


$$
\begin{aligned}
& P_{i}\left(Y=1 \mid X_{1}, X_{2}, \ldots, X_{k}\right)=F\left(\beta_{0}+\beta_{1} X_{1}+\beta_{2} X_{2}+\ldots+\beta_{k} X_{k}\right) \\
& P_{i}\left(Y=1 \mid X_{1}, X_{2}, \ldots, X_{k}\right)=\frac{1}{1+e^{-\left(\beta_{0}+\beta_{1} X_{1}+\beta_{2} X_{2}+\ldots+\beta_{k} X_{k}\right)}}
\end{aligned}
$$

Onde $\mathrm{P}_{\mathrm{i}}$ é a probabilidade de uma empresa ter operações de fusão e aquisição e $\beta$ os parâmetros a serem estimados e $e$ a base dos logaritmos naturais.

Pela natureza não linear da transformação logística, os coeficientes do modelo logit são estimados por máxima verossimilhança. Afinal, a regressão logística maximiza a probabilidade de que um evento aconteça (HAIR JR. et al, 2009).

Desta forma a probabilidade de a empresa não ter passado por operações de F\&A é medida por:

$$
\left(1-P_{i}\right)=\frac{1}{1+e^{\left(\beta_{0}+\beta_{1} X_{1}+\beta_{2} X_{2}+\ldots+\beta_{k} X_{k}\right)}}
$$

Onde a razão de probabilidade (odds ratio) é dada por:

$$
\begin{aligned}
\frac{P_{i}}{\left(1-P_{i}\right)} & =\frac{1+e^{\left(\beta_{0}+\beta_{1} X_{1}+\beta_{2} X_{2}+\ldots+\beta_{k} X_{k}\right)}}{1+e^{-\left(\beta_{0}+\beta_{1} X_{1}+\beta_{2} X_{2}+\ldots+\beta_{k} X_{k}\right)}} \\
& =e^{\left(\beta_{0}+\beta_{1} X_{1}+\beta_{2} X_{2}+\ldots+\beta_{k} X_{k}\right)}
\end{aligned}
$$

Logaritmizando a expressão, tem-se:

$$
\ln \left(\frac{P_{i}}{1-P_{i}}\right)=\beta_{0}+\beta_{1} X_{1}+\beta_{2} X_{2}+\ldots+\beta_{k} X_{k}
$$

o que indica que os coeficientes estimados medem o impacto da variação da variável explicativa no logaritmo da razão da probabilidade de que as empresas realizem ou não processos de F\&A.

Como já afirmado, neste modelo, a variável dependente assume valores binários ( 0 e 1$)$ onde o acontecimento de uma operação de F\&A assume valor 1 e a não ocorrência valor 0 . As variáveis independentes são compostas pelas variáveis que representam as características econômico-financeiras da empresa (Figura 1).

Como as probabilidades são desconhecidas, a estimação do modelo, como mencionado, se deu pelo método da máxima verossimilhança, pois este seleciona os coeficientes que tornam os valores observados mais prováveis de terem ocorrido. Esta estatística é chamada de log-verossimilhança e de acordo com Field (2009) é dada pela seguinte expressão: $\log -$ verossimilhança $=\sum_{i=1}^{N}\left\{Y_{i} \ln \left(P\left(Y_{i}\right)+\left(1-Y_{i}\right) \ln \left[1-P\left(Y_{i}\right)\right]\right\}\right.$

Para fins de adequação do modelo foi verificada, por meio do teste de correlação de Person, a presença de multicolinearidade entre as variáveis. $\mathrm{O}$ valor elevado do coeficiente de correlação é um dos indicadores de presença de multicolinearidade. Para Hair Jr. et al. (2009) coeficientes com valores acima de 0,60 pode indicar esse tipo de problema, ou seja, as variáveis podem apresentar algum tipo de relação que poderia impedir a mensuração do modelo.

Contudo, para Alisson (2003), examinar a matriz de correlação não é suficiente para identificar os problemas de multicolinearidade. Então Hair Jr. et al. (2009) sugerem que o fator de inflação da variância (VIF) e a tolerância (TOL) sejam verificados. Para os autores, valores do VIF acima de 10 e de TOL abaixo de 0,1 indicam sérios problemas de multicolinearidade. Desta forma quando as variáveis do modelo apresentam valores de TOL e VIF aceitáveis, pode-se afirmar que o modelo logit será estimado com confiança.

Ademais, Hair Jr. et al (2009), afirmam que é necessário testar a aderência do modelo com o teste de Hosmer e Lemeshow, que testa a hipótese de que os dados observados são significativamente diferentes dos valores previstos pelo modelo. Em sequência, a verificação de presença de autocorrelação nos resíduos foi feita por meio do teste de Durbin Watson. Gujarati (2006) afirma que para os valores do teste localizados entre 2 e 4, deve-se aceitar a hipótese nula de que o modelo não apresenta nenhum tipo de autocorrelação, quer positiva ou negativa.

Assim, os dados foram tratados utilizando o pacote estatístico Statistical Package for the Social Science (SPSS ${ }^{\circ}$ ). Ademais uma vez que o objetivo deste trabalho se finda em identificar as características econômico-financeiras das empresas que foram determinantes para os processos de F\&A, os dados foram trabalhados com defasagem de um ano, isto é, para as operações de fusão e aquisição do ano $t$, utilizou-se os as variáveis referentes ao ano $t$-1. Desta forma, pode-se captar se estas são determinantes nos processos de F\&A. 


\subsection{Hipóteses e composição das variáveis}

Como apresentado anteriormente, o modelo teórico deste trabalho foi definido por características econômico-financeiras das empresas. A partir destas características, consideradas determinantes para a ocorrência dos processos de F\&A, as hipóteses deste trabalho foram estabelecidas, conforme podem ser observadas no Quadro 5.

Quadro 5 Construção das hipóteses

\begin{tabular}{|c|c|c|}
\hline & Características das empresas & Hipóteses \\
\hline Eficiência operacional & $\begin{array}{l}\text { Andrade e Stafford (2004) afirmam que a empresa adquirente normalmente } \\
\text { é mais eficiente. Para os autores, quanto melhor os índices de endivida- } \\
\text { mento e condições operacionais favoráveis, mais fácil será para a empresa } \\
\text { adquirente absorver as operações da empresa alvo. Afinal se as empresas } \\
\text { que apresentarem baixo nível de aproveitamento dos ativos para geração } \\
\text { de caixa pode indicar que os gestores da empresa realizaram investimentos } \\
\text { que não resultam em retornos positivos. Assim, a partir do momento que a } \\
\text { empresa apresenta eficiência operacional, a capacidade instalada e a aloca- } \\
\text { ção de recursos da mesma pode ser melhor aproveitado, levando a empresa } \\
\text { a optar por processos de F\&A. }\end{array}$ & $\begin{array}{l}\text { Hipótese 1: quanto maior a eficiên- } \\
\text { cia operacional da empresa, maior } \\
\text { a probabilidade da mesma realizar } \\
\text { operações de F\&A. }\end{array}$ \\
\hline Estrutura de capital & $\begin{array}{l}\text { Camerlynck, Ooghe e Langhe (2005) afirmam que as empresas se tornam } \\
\text { alvo de fusões e aquisições pelo fato de estarem endividadas e veem como } \\
\text { alternativa à falência a participação nos processos de fusões e aquisições. } \\
\text { Assim, para Adelaja, Nayga e Farooq (1999) as empresas que possuem um } \\
\text { percentual de capital próprio menor que o capital de terceiros são mais } \\
\text { propensas a se tornarem adquirentes nos processos de F\&A. }\end{array}$ & $\begin{array}{l}\text { Hipótese 2: quanto maior a parti- } \\
\text { cipação de capital de terceiros na } \\
\text { empresa, maior a probabilidade de } \\
\text { realizar operação de F\&A. }\end{array}$ \\
\hline Liquidez & $\begin{array}{l}\text { Camerlynck, Ooghe e Langhe (2005), afirmam que as empresas adquiren- } \\
\text { tes tendem a apresentar baixos índices de liquidez, pois supõem que as } \\
\text { empresas alvo tem bons índices de liquidez e que quando associados com a } \\
\text { adquirente, resultará em ganhos para esta última. }\end{array}$ & $\begin{array}{l}\text { Hipótese 3: quanto maior a liquidez } \\
\text { da empresa, menor a probabilidade } \\
\text { de realizar operação de F\&A. }\end{array}$ \\
\hline Rentabilidade & $\begin{array}{l}\text { A rentabilidade de uma empresa pode sofrer influencia de seus administra- } \\
\text { dores. Desta forma, Gorton, Kahl e Rosen (2009) colocam que se identificado } \\
\text { que a empresa está sendo gerenciada de forma ineficiente, e outra empresa } \\
\text { ter ciência disto, pode tornar a empresa alvo de fusão e aquisição, isto é, se } \\
\text { interessar pela aquisição da empresa. }\end{array}$ & $\begin{array}{l}\text { Hipótese 4: quanto maior a } \\
\text { rentabilidade da empresa, maior a } \\
\text { probabilidade dela realizar opera- } \\
\text { ções de F\&A. }\end{array}$ \\
\hline Retorno ao acionista & $\begin{array}{l}\text { Meglio e Risberg (2010) afirmam que em uma operação de F\&A, um dos } \\
\text { pontos a se considerar é a criação de valor ao acionista. Já Alcade e Espitia } \\
\text { (2003) pontuam que alguns estudos demonstram conclusivamente que } \\
\text { os acionistas das empresas alvo e adquirente têm retornos positivos com a } \\
\text { ocorrência da operação de F\&A. }\end{array}$ & $\begin{array}{l}\text { Hipótese 5: quanto maior a criação } \\
\text { de valor ao acionista, maior a } \\
\text { probabilidade de realizar operações } \\
\text { de F\&A. }\end{array}$ \\
\hline Tamanho & $\begin{array}{l}\text { O tamanho da empresa segundo Gorton, Kahl e Rosen (2009) é um fator } \\
\text { que determina se ela será alvo ou adquirente nas operações de fusão e } \\
\text { aquisição. Os autores afirmam que as empresas pequenas não são alvos de } \\
\text { fusões e aquisições, contudo as empresas adquirentes são, na maioria dos } \\
\text { casos, maiores que as empresas alvo. }\end{array}$ & $\begin{array}{l}\text { Hipótese 6: quanto maior o } \\
\text { tamanho das empresas, maior a } \\
\text { probabilidade de realizar operações } \\
\text { de F\&A. }\end{array}$ \\
\hline
\end{tabular}

Estas características das empresas são representadas por variáveis que indicam a situação econômico-financeira das empresas, as quais permitem avaliar as hipóteses estabelecidas. Para tal, o Quadro 6 apresenta a forma como cada uma das variáveis foram do modelo teórico (Figura 1) foram calculadas. 
Thaís Alves dos Santos • Cristina Lelis Leal Calegario - Francisval de Melo Carvalho

Quadro 6 Cálculo dos indicadores econômico-financeiros

\begin{tabular}{|c|c|c|}
\hline Índice & Cálculo & Representa \\
\hline Giro do ativo (GA) & $\frac{\mathrm{RLO}}{\mathrm{A}}$ & $\begin{array}{l}\text { Quanto a empresa vendeu em relação ao } \\
\text { investimento. }\end{array}$ \\
\hline Composição do endividamento (CE) & $\frac{P C}{C T}$ & $\begin{array}{l}\text { O percentual de obrigações a curto prazo } \\
\text { em relação as obrigações totais. }\end{array}$ \\
\hline Imobilização do patrimônio líquido (IPL) & $\frac{\mathrm{AP}}{\mathrm{PL}}$ & $\begin{array}{l}\text { Quanto a empresa aplicou no AP em relação } \\
\text { do PL. }\end{array}$ \\
\hline Imobilização de recursos (IRC) & $\frac{A P}{P L+E L P}$ & O percentual de PL e ELP foi destinado ao AP. \\
\hline Liquidez geral (LG) & $\frac{A C+R L P}{P L+E L P}$ & $\begin{array}{l}\text { Quanto a empresa possui de AC e RLP em } \\
\text { relação as dívidas totais. }\end{array}$ \\
\hline Liquidez corrente (LC) & $\frac{A C}{P L}$ & $\begin{array}{l}\text { Quanto de AC a empresa possui em relação } \\
\text { ao PC. }\end{array}$ \\
\hline Liquidez seca (LS) & $\frac{A C-\text { estoques }}{P L}$ & Quanto de ativo líquido em relação ao PC. \\
\hline Rentabilidade do ativo (ROA) & $\frac{\mathrm{LL}}{\mathrm{A}}$ & $\begin{array}{l}\text { Quanto a empresa tem de lucro em relação } \\
\text { aos investimentos. }\end{array}$ \\
\hline Retorno sobre o capital próprio (ROE) & $\frac{\mathrm{LL}}{\mathrm{PL}}$ & $\begin{array}{l}\text { Quanto a empresa tem de lucro em relação } \\
\text { ao capital próprio. }\end{array}$ \\
\hline Margem operacional (MO) & $\frac{\mathrm{LL}}{\mathrm{RLO}}$ & $\begin{array}{l}\text { Quanto a empresa obtém de lucro em } \\
\text { relação às vendas. }\end{array}$ \\
\hline Valor econômico adicionado (EVA) & $\mathrm{LL}-(\mathrm{PL} \times \mathrm{CDI})$ & $\begin{array}{l}\text { Quanto a empresa criou ou destruiu de valor } \\
\text { para seus acionistas. }\end{array}$ \\
\hline \multicolumn{3}{|l|}{ Onde: } \\
\hline A: ativos totais & AC: ativo circulante & AP: ativo permanente \\
\hline CDI: taxa livre de risco & CT: capital de terceiros & ELP: exigível longo prazo \\
\hline LL: Iucro líquido & PC: passivo circulante & PL: patrimônio líquido \\
\hline RLP: realizável longo prazo & RLO: Recita líquida operacional & \\
\hline
\end{tabular}

Fonte: Matarazzo (1998); Braga, Nossa e Marques (2004); Padoveze (2004) 


\section{RESULTADOS}

Para identificar as características econômico-financeiras determinantes dos processos de F\&A sob a ótica das empresas adquirentes, o modelo de regressão logística utilizado foi:

$F \& A=\beta_{0}+\beta_{G A} G A+\beta_{C 3} C 3+\beta_{C E} C E+\beta_{I P L} I P L+\beta_{I B C} I R C+\beta_{I G} L G+$ $\beta_{L C} L C+\beta_{L L} L S+\beta_{R O A} R O A+\beta_{R O E} R O E+\beta_{M O} M O+\beta_{E V A} E V A+\beta_{A T} A T$

Onde as características da empresa são representadas pelas variáveis: giro do ativo (GA), capital de terceiros (C3), composição do endividamento (CE), imobilização do patrimônio líquido (IPL), imobilização de recursos (IRC), liquidez geral (LG), liquidez corrente (LC), liquidez seca (LS), rentabilidade do ativo (ROA), retorno sobre o capital próprio (ROE), margem operacional (MO), valor econômico adicionado (EVA) e tamanho da empresa (AT).

A partir da equação (1) realizou-se a verificação da aderência do modelo. O teste de Hosmer e Lemeshow indicou que o modelo tem aderência, isto é, pode ser estimado. Já o teste de Durbim Watson, indicou que não há presença de autocorrelação nos resíduos do modelo. Em seguida o cálculo do modelo de regressão logística foi realizado, com um limite de confiança de 95\%, confirmando seu ajuste satisfatório. Ademais, $\mathrm{O}$ teste qui-quadrado foi significativo a $1 \%$ indicando que o modelo se ajustou de forma satisfatória aos dados a uma porcentagem de 90,8.

Os resultados da regressão logística são apresentados na Tabela 1. Observando os resultados da regressão logística identifica-se que as variáveis GA, CE, ROA, EVA e AT foram significativas na equação 1. Todavia dentre estas variáveis, quatro apresentam sinal positivo em seus coeficientes, isto é, estas variáveis exercem influência sobre os processos de F\&A, logo podem ser consideradas como fatores determinantes para sua ocorrência. Essas variáveis foram: GA, ROA, EVA e AT.

O primeiro fator determinante das operações de F\&A, GA, é um índice que relaciona o total das vendas produzidas com os ativos da empresa, ou seja, mostra o quão eficiente a empresa se apresentou no período. Este resultado corrobora o trabalho de Andrade e Stafford (2004) quando estes autores afirmam que empresas eficientes, normalmente são as adquirentes. Confirmando assim a hipótese 1 que afirma que a eficiência operacional positiva influencia as operações de F\&A. Como estas operações tem dispêndios financeiros que serão diluídos ao longo dos anos, uma empresa que apresenta baixa eficiência operacional, poderia, caso entre em um processo de F\&A, chegar a um resultado não satisfatório, por já apresentar dificuldades anteriormente ao processo de F\&A.

Das variáveis que representam a estrutura de capital das empresas, a única significativa, foi a $\mathrm{CE}$, contudo o sinal que apresentado pela variável indica que ela não causa impactos nas operações de F\&A. Assim pode-se concluir que estrutura de formação do capital da empresa não é determinante para as operações F\&A.

Do conjunto de variáveis que representa a rentabilidade das empresas, a rentabilidade do ativo, ROA, foi a única que se confirmou determinante nos processos de F\&A. Afinal, como colocado por Gorton, Kahl e Rosen (2009), se uma empresa apresenta baixa rentabilidade, pode ser indício de que a mesma está sendo mal gerenciada. Especificamente o ROA positivo indica que os ativos da empresa estão sendo aplicados com eficiência e que os mesmos estão gerando lucros positivos. O que por sua vez, contribui para o crescimento da empresa e dá subsídios para que esta realize uma operação de F\&A.

A variável EVA apresentou resultado positivo, o que indica que da empresa estão sendo beneficiados com os investimentos da empresa. Meglio e Risberg (2010) afirmaram que a criação de valor ao acionista é crucial para o sucesso de uma operação de F\&A. Assim se o retorno ao acionista apresenta resultados positivos, pode-se considerar que a empresa tem maior probabilidade de realizar um processo de $\mathrm{F} \& \mathrm{~A}$, com o objetivo de maximizar os ganhos com sinergias operacionais e os ganhos de seus acionistas.

Ismail e Krause (2010) já afirmavam que o tamanho das empresas é relevante na determinação dos processos de F\&A. Este trabalho corrobora com os autores, pois a relação positiva do tamanho da empresa com a variável dependente, aponta que este é determinante nos processos de F\&A. Assim considera-se que quanto maior a empresa, maiores as chances de que esta realize em um processo de F\&A. 
Tabela 1 Modelo 1 de regressão logística dos fatores que determinam as operações de F\&A

\begin{tabular}{|c|c|}
\hline & Coeficiente/significância \\
\hline Constante & $-10,0^{* * *}$ \\
\hline GA: Giro do Ativo & $694,3^{* * *}$ \\
\hline CE: Composição do Endividamento & $-1655,6^{* * *}$ \\
\hline IPL: Imobilização do Patrimônio Líquido & $-21,4^{\text {n.s. }}$ \\
\hline IRC: Imobilização de Recursos & $0,3^{\text {n.s. }}$ \\
\hline LG: Liquidez Geral & $-73,3^{\text {n.s. }}$ \\
\hline LS: Liquidez Seca & $-2,4^{\text {n.s. }}$ \\
\hline MO: Margem Operacional & $0,5^{\text {n.s. }}$ \\
\hline ROA: Rentabilidade do Ativo & $0,2^{* *}$ \\
\hline ROE: Retorno sobre o Capital Próprio & $-84,8^{\text {n.s. }}$ \\
\hline EVA: Valor Econômico Adicionado & $0,0^{* * *}$ \\
\hline AT:Tamanho & $0,7^{7 * * x}$ \\
\hline$x_{2}$ & 371,304 \\
\hline \% Concordância & $93,1 \%$ \\
\hline R2 Cox \& Snell & $9,3 \%$ \\
\hline R2 Nagelkerke & $23,8 \%$ \\
\hline-2 Log likelihood & $1.512,449$ \\
\hline
\end{tabular}

Destarte, pode-se afirmar que com base nos resultados da equação (1) que as características da empresa que são determinantes das operações de F\&A são a eficiência operacional, rentabilidade, retorno ao acionista e o tamanho da empresa.

Consequentemente foi possível confirmar/refutar as hipóteses de estudo estabelecidas. A primeira hipótese confirmada, se refere a eficiência operacional da empresa, pois a variável a representa foi significativa e positiva. A próxima hipótese confirmada foi a quarta, onde considera-se que empresas com boa rentabilidade tem mais chances de realizarem operações de F\&A. A quinta hipótese, que trata do retorno ao acionista das empresas adquirente, também pôde ser confirmada. Como a variável desta hipótese também se apresentou significativa e positiva, considera-se então que o retorno ao acionista é determinante para a realização das operações de F\&A.

A relação positiva e significativa do tamanho da empresa com as operações de F\&A, permite confir- mar a sétima hipótese. Ou seja, empresas maiores, são mais propensas a realizarem processos de F\&A. Por fim, aquelas hipóteses que apresentaram significância estatística e sinal negativo e/ou não apresentarem significância, foram refutadas, isto é, as hipóteses 2 e 3. O quadro 7 apresenta uma síntese das hipóteses confirmadas/refutadas.

É interessante ressaltar que a forma de financiamento da empresa não se mostrou determinante das operações de F\&A, pois a variável CE apesar de significativa apresentou coeficiente negativo, diferindo assim do que foi colocado por Adelaja, Nayga e Farooq (1999), quando os autores falam que empresas com percentuais de capital próprio menor que o capital de terceiros, podem ser caracterizadas como possíveis adquirentes. Diferente da CE, as variáveis IPL e IRC não foram significativas, sendo que a IPL ainda apresentou coeficiente negativo. Desta forma a hipótese de que a estrutura de capital é determinante das operações de F\&A não pode ser confirmada. 
Quadro 7 Hipóteses - resultados

\begin{tabular}{c|c}
\hline Hipótese & Condição \\
\hline$H_{1}$ & Confirmada \\
\hline$H_{2}$ & Refutada \\
\hline$H_{3}$ & Refutada \\
\hline$H_{4}$ & Confirmada \\
\hline$H_{5}$ & Confirmada \\
\hline$H_{6}$ & Confirmada \\
\hline
\end{tabular}

Assim como as variáveis que representam a estrutura de capital das empresas, as variáveis que representam a liquidez das empresas apresentaram resultados negativos e não significativos. Logo, apesar de Camerlynck, Ooghe e Langhe (2005), afirmarem que as empresas adquirentes tendem a apresentar baixos índices de liquidez, os resultados não permitem confirmar a hipótese de que a liquidez das empresas é um fator determinante das operações de F\&A.

\section{CONCLUSÕES}

Apesar dos constantes períodos de incertezas que as economias mundiais passam, é notório o crescimento das operações de F\&A. Estas têm sido vistas como uma alternativa das empresas para entrada em novos mercados e expansão da empresa no mercado interno.

Visto o crescimento destas operações, o objetivo deste trabalho foi identificar quais os fatores determinantes das operações de F\&A na Indústria Manufatureira. Para tal trabalhou-se com dados econômico-financeiros das empresas.

Apesar de algumas variáveis não terem apresentados resultados significativos, acredita-se que o objetivo do estudo foi alcançado. Algumas variáveis, ou seja, características da empresa se mostraram significativas aos acontecimentos das operações de F\&A.

Foram estabelecidas seis hipóteses baseadas em um conjunto de variáveis. A primeira hipótese estabelecida foi confirmada, ou seja, a eficiência operacional das empresas pode ser considerada como fator determinante das operações de F\&A. Diferente da primeira, a segunda hipótese, que trata da estrutura de capital da empresa não pode ser confirmada, apesar de uma de suas variáveis apresentar resultados significativos. As variáveis que apresentaram resultados significativos negativos, mostraram que aquela variável específica não é determinante para o acontecimento das operações de F\&A, contudo elas influenciam as operações de F\&A.

A terceira hipótese também não foi confirmada. Esta que tratava da liquidez da empresa, ou seja, a capacidade de pagamento das empresas, a capacidade das empresas converterem seus ativos em monetário, não apresentou significância em suas variáveis. A próxima hipótese, $\mathrm{H}_{4}$, foi confirmada. A rentabilidade de uma empresa representa a remuneração do capital investido, desta forma uma empresa com boa rentabilidade, implica em uma empresa cujos recursos estão sendo bem aplicados. Assim como vários estudos nacionais e internacionais afirmam que a rentabilidade das empresas influencia nas operações de F\&A, este trabalho comprovou que a rentabilidade pode ser considerada um fator determinante destas operações.

A quinta hipótese, que trata do retorno ao acionista também foi confirmada. Corroborando a literatura, a qual afirma que o retorno ao acionista é fator determinante para o acontecimento das operações de F\&A. Afinal, um dos motivadores das decisões por operações de F\&A é o ganho ao acionista das empresas adquirentes. Por fim, a sexta hipótese, relacionada ao tamanho da empresa, também foi confirmada. Demonstrando que se a empresa apresenta maior porte, tem maior probabilidade de realizar operações de F\&A.

A ótica sob a qual este trabalho se estabelece, é nas empresas consideradas adquirentes, ou seja, as que não foram alvo das operações de F\&A. Assim, os resultados deste trabalho mostram que os fatores determinantes das operações de fusão e aquisição são a eficiência operacional, rentabilidade, retorno ao acionista e tamanho. Os resultados deste trabalho são específicos para a Indústria Manufatureira, não podendo ser generalizados.

Assim, sugere-se como agenda de pesquisa a inclusão de variáveis macroeconômicas que captem a influencia do ambiente externo nas operações de F\&A. Outra sugestão proposta é comparar os determinantes destas operações no período após a 
nova regulamentação do Cade, visto que, mesmo com as novas exigências do regulamento, as operações de F\&A continuam crescendo no mercado. Investigar sobre a influência destes processos na concentração de mercado e seus efeitos na concorrência também se faz válido.

\section{REFERÊNCIAS}

ADELAJA, A; NAYGA JR., R.; FAROOQ, Z. Predicting mergers and acquisitions in the food industry. Agribusiness, Vol. 15, N. 1, 1-23. 1999.

ALISSON, P. D. Logistic regression using the SAS system: theory and application. Cary: SAS Institute, 2003. 288p.

ANDRADE, G.; STAFFORD, E. Investigating the economic role of mergers. Journal of Corporate Finance, Boston, v. 10, p. 1-36, 2004.

BARNES, P. Can Takeover targets be identified by statistical techniques?: some UK evidence. Journal of the Royal Statistical Society. Series D (The Statistician), Vol. 47, no. 4, pp. 573-591, 1998.

BARNES, P. Predicting UK takeover targets: some methodological issues and an empirical study. Review of Quantitative Finance and Accounting. The Netherlands. Vol. 12, no 3, 1999.

BAUGUESS, S.W.; MOELLER ，S. B.; SCHLINGEMANN, F. P.; ZUTTER, C. J. Ownership structure and target returns. Journal of Corporate Finance, v. 15, pp. 48-65, 2009.

BORTOLUZZO, A. B.; GARCIA, M. P. S.; BOEHE, D. M.; SHENG, H. H. Desempenho de fusões e aquisições cross border: análise empírica do caso brasileiro. Revista de Administração de empresas, volume 51, n. 6, p. 659-671, nov-dez 2014.
BRAGA, R.; NOSSA, V.; MARQUES, J. A. V. C. Uma proposta para a análise integrada da liquidez e rentabilidade das empresas. Revista Contabilidade e Finanças - USP. Edição especial, p.51-64, 30 de junho de 2004.

BRASIL. Lei Federal no 6404. Brasília: Congresso Nacional, 1976. D.O. DE 17/12/1976, P. 1 (SUPLEMENTO)

BRITO, G. A. S., BATISTELlA, F. D., FAMA, R. Fusões e Aquisições no Setor Bancário: Avaliação Empírica do Efeito Sobre o Valor das Ações. RAUSP - Revista de Administração da Universidade de São Paulo, São Paulo, v.40, n.4, p.353-360, out./nov./ dez. 2005.

CAMARGOS, M. A.; BARBOSA, F. V. Análise do desempenho econômico-financeiro e da criação de sinergias em processos de fusões e aquisições do mercado brasileiro ocorridos entre 1995 e 1999. Caderno de Pesquisas em Administração, São Paulo, v. 12, n.2, p. 99-115, abril/junho 2005.

CAMARGOS, M. A.; BARBOSA, F. V. Fusões e aquisições de empresas no brasileiras: criação de valor e sinergias operacionais. RAE. V 49, n2, pág 206-220, abr./jun. 2009.

CAMARGOS, M. A.; BARBOSA, F. V. Fusões e Aquisições em empresas Brasileiras: Sinergias Operacionais, Gerenciais e Rentabilidade. Contabilidade Vista \& Revistas, vol.21, n.1. 2010.

CAMARGOS, Marcos A.; BARBOSA, Francisco V. Fusões, Aquisições e Takeovers: um levantamento teórico dos motivos, hipóteses testáveis e evidências empíricas. Caderno de Pesquisas em Administração, São Paulo, v. 10, n. 2, p.17-38, abril/junho, 2003.

CHENERY, H. B. Patterns of Industrial Growth. The American Economic Review, Vol. 50, No. 4, 624654, 1960. 
CLEMENTS, M.; SINGH, H. An analysis of trading in target stocks before successful takeover announcements. Journal of Multinational Financial Management, v. 21, pp. 1-17, 2011.

DENČIĆ-MIHAJLOV, K.; RADOVIĆ, O. Problems in predicting target firms at the undeveloped capital markets. Facta Universitatos. Series: Economics and Organization Vol. 3, No 1, 2006, pp. 59 - 68.

DHAWAN, S. Mergers and Acquisitions in Global Context. Kindler, vol. 4, n.1, pp.7-16, 2009.

DUCHIN, R.; SCHMIDT, B. Riding the merger wave: uncertainty, reduced monitoring and bad acquisitions. Journal of Financial Economics, v. 107, p. 69-88, 2013.

FIELD, A. Descobrindo a estatística usando o SPSS. Porto Alegre: Artmed, 2009.

FRANCO, P. M.; CAMARGOS, M. A. Fusões e aquisições no setor bancário brasileiro: criação de valor, rentabilidade, sinergias operacionais e grau de concentração. Anais XXXV Encontro da Anpad. Rio de Janeiro/RJ, 4 a 7 de setembro de 2011.

GAUGHAN, P. A. Mergers, acquisitions and corporate restructurings, 5 ed. New Jersey: John Wiley \& Sons, Inc., 2011.

GORTON, G.; KAHL, M.; ROSEN, R. J. Eat or be eaten: a theory of mergers and firm size. The Journal of Finance. V. 64, issue 3, pages 1291-1344, june 2009.

GUJARATI, D. Econometria Básica. $4^{\mathrm{a}}$ ed. Elsevier: Rio de Janeiro, 2006.

HAIR JR., J. F.; BLACK, W. C.; BABIN, B, J.; ANDERSON, R. E.; TATHAM, R. L. Análise Multivariada de Dados. $6^{\mathrm{a}}$ ed. Porto Alegre: Bookman, 2009.

HSU, K. C.; WRIGHT, M.; ZHU, Z. What motivates merger and acquisition activities in the upstream oil \& gas sector in the U.S.? Energy economics. Vol. 65, June 2017, pages 240-250.
HUNJURA, A. I.; ABBAS, Q.; SAEED, R.; HASSAN, E. U.; IJAZ, M. A. Analysis of pre and post merger and acquisition: financial performance of banks in Pakistan. Information Management and Business Review. Vol. 6, No. 4, pp. 177-190, Aug 2014.

IBGE. Pesquisa Industrial. Rio de Janeiro, v. 29, n.1, p.1-182, 2010

IBGE. Pesquisa Industrial, Rio de Janeiro, v. 35, n. 1, p. 1-44, 2016

ISMAIL, A.; KRAUSE, A. Determinants of the method of payment in mergers and acquisitions. The Quaterly Review of Economics and Finance. Volume 50, issue 4, november 2010, pages 471-484.

KING, D. R.; DALTON, D. R. CATHERINE, M. D; COVIN, J. G. Meta-Analyses of post-acquisition performance: indicators of unidentified moderators. Strategic Management Journal. 25: 187-200. 2004.

KLOECKNER, G. O. Fusões e aquisições: motivos e evidência empírica. Revista de Administração. São Paulo: FEA/USP, v. 29, n.1, p.42-58, Jan./Mar. 1994.

KPMG Corporate Finance. Pesquisa de fusões e aquisições 2011 - 4º trimestre. 2012.

KPMG Corporate Finance. Pesquisa de fusões e aquisições 2017 - $4^{\circ}$ trimestre. 2018

KUPFER, D.; HASENCLEVER, L. Economia Industrial. Rio de Janeiro: Elsevier, 2002.

MATARAZZO, D. C. Análise Financeira de Balanços - Abordagem Básica e Gerencial. 5 ed. São Paulo: Atlas, 1998. $471 \mathrm{p}$.

MEGLIO, O.; RISBERG, A. Mergers and acquisitions - Time for a methodological rejuvenation of the field? Scandinavian Journal of Management, $\mathrm{v}$. 86 , p. $87-95,2010$.

MIRANDA, J. C.; MARTINS, L. Fusões e aquisições de empresas no Brasil. Economia e Sociedade. Campinas, v. 14, pág 67-88, jun. 2000. 
NARDI, R. Y. S.; SILVA, E. S. Criação de Valor em Fusões e Aquisições: A Influência do Sentimento de Mercado. XXXVI Encontro da Anpad. Rio de Janeiro/RJ, 22 a 26 de setembro de 2012.

ÖBERG, C.; HOLTSTRÖM, J. Are mergers and acquisitions contagious? Journal of Business Research 59 (2006) 1267-1275

PADOVEZE, C. L. Análise das demonstrações financeiras. Cengage Learning Editores, 2004, 267p.

PALEPU, K. Predicting Takeover targets: a methodological and empirical analysis. Journal of Accountingand Economics, v. 8, pp. 3-35, 1986.

PARK, K.; JANG, S. S. Mergers and acquisitions and firm growth: investigating restaurant firms. International Journal of Hospitality Management. Volume 30, issue 1, march 2011, pages 141-149

PATROCÍNIO, M. R.; KAYO, E. K.; KIMURA, H. Aquisição de empresas, intangibilidade e criação de valor: um estudo de evento. RAUSP - Revista de Administração da Universidade de São Paulo, São Paulo, v.42, n.2, p.205-215, abr./maio/jun. 2007

PEREIRA, C. M. L.; DORNELAS, J. S. Fatores Promotores e Inibidores do Alinhamento Estratégico da Tecnologia da Informação em uma Situação de Fusão: o Caso de uma Rede Varejista. RAC, Curitiba, v. 14, n. 3, art. 6, pp. 495-515, Mai./Jun. 2010.

PESSANHA, G. R. G. Estudos empíricos de fusões e aquisições no setor bancário brasileiro (Tese). Universidade Federal de Lavras, 2016.

PESSANHA, G. R. G.; CALEGARIO, C. L. L.; SÁFADI, T.; ÁZARA, L. N. Impactos das estratégias de fusão e aquisição na rentabilidade dos bancos adquirentes: uma aplicação dos modelos de intervenção no setor bancário brasileiro. Revista de Administração Mackenzie. v. 13, n.5, p.101-134. Set./Out. 2012a.
PESSANHA, G. R. G.; SANTOS, T. A.; ALCÂNTARA, J. N.; CALEGARIO, C. L. L. Influências das fusões e aquisições no valor de mercado das instituições bancárias adquirentes: uma aplicação da metodologia de estudo de eventos no período de 1994 a 2009. XV SemeAd, outubro 2012b.

PIMENTEL, R. F. Gestão, estratégia e considerações sobre a nova teoria da firma. Universidade Federal Fluminense, 2004.

ROSS, S.; WESTERFIELD, R. W.; JAFFE, J. F. Administração financeira. 2. ed. São Paulo: Atlas, 2007. 776p.

ROSSI, S.; VOLPIN, P. Cross-country determinants of mergers and acquisitions. ECGI - Finance Working Paper No. 25/2003; AFA 2004 San Diego Meetings; EFA 2003 Annual Conference Paper No. 251

SCHERER, F. M. Industrial market structure and economic performance. Chicago: Rand McNally, 1970.

SILVA, E. S.; KAYO, E. K.; NARDI, R. Y. S. Governança corporativa e desempenho em aquisições de empresas. XXXVI Encontro da ANPAD. Rio de Janeiro/ RJ. 22 a 26 de setembro de 2012.

STOCK, J. H.; WATSON, M, W. Econometria. São Paulo: A. Wesley, 2004, 485 p.

TOIGO, L. A.; HEIN, N. Desempenho das Companhias Pós-Fusões e Aquisições Mensurado pelos Filtros de Graham. Revista Catarinense da Ciência Contábil, v. 16, n. 49 , p. $34-50,2017$.

TRICHES, D. Fusões, aquisições e outras formas de associação entre empresas no Brasil. Revista de Administração, São Paulo, v.31, n.1, p. 14-31, jan./ mar. 1996.

VIEIRA, F. V.; AVELALR, A. P.; VERÍSSIMO, M. P. Indústria e crescimento econômico: evidências para países desenvolvidos e em desenvolvimento. Revista de Economia Política, vol. 34, nº 3 (136), pp. 485-502, julho-setembro/2014. 
WESTON, J. F.; SIU, J. A.; JOHNSON, B. A. Takeovers, restricting, \& corporate governance, 5.ed. New Jersey: Printice Hall, 2006.

WOOD JR, T.; VASCONCELOS, F. C.; CALDAS, M. P. Fusões e aquisições no Brasil. Revista de Administração de Empresas Executivo, São Paulo, v. 2 , n. 4 , nov. $2003 /$ jan 2004.
ZILBER, M. A.; FICHMANN, A. A.; PIKIENY, E. Alternativas de crescimento: a alternativa de fusões e aquisições. Revista de Administração Mackenzie. Ano 3, n.2, p.137-154. 2002. 